\title{
Embeddings of $U(1)$-Current Algebras in Non-Commutative Algebras of Classical Statistical Mechanics *
}

\author{
Alain Connes ${ }^{1}$ and David E. Evans ${ }^{2}$ \\ ${ }^{1}$ Institut des Hautes Études Scientifiques, 35 Route de Chartres, \\ F-91440 Bures-sur Yvette, France \\ ${ }^{2}$ Department of Mathematics and Computer Science, University College of Swansea, \\ Singleton Park, Swansea SA2 8PP, Wales, UK
}

\begin{abstract}
Motivated by multiplicative $K$-homology, and understanding critical phenomena in some classical statistical mechanical models, we construct actions of $G L(\infty)$ on the operator algebras of V. Jones and Ocneanu, and analyse these in terms of embeddings of $U(1)$-current algebras.
\end{abstract}

\section{Introduction}

In this paper we shall describe a construction of actions of the group $G L(\infty)$ on the $\mathrm{V}$. Jones and Ocneanu algebras, to which both authors of this paper arrived at independently and for different reasons.

The first author was motivated by the search for a multiplicative analogue of the known additive $K$-homology theory. In this the Jones' index of subfactors would be the multiplicative analogue of the codimension of a subspace of a Hilbert space. This analogy would be in the same way as the classification of outer conjugacy classes of automorphisms of the hyperfinite factor of type $\mathrm{II}_{1}$ is the multiplicative analogue of the classification of unitaries modulo the compact operators. The basic notion of a Fredholm module $(\mathfrak{h}, D)$ on an algebra $\mathfrak{A}$ gets replaced by a pair $\left(A, \sigma_{t}\right)$ where $A$ is a $C^{*}$-algebra, $\sigma_{t}$ a one parameter group of automorphisms, while the unitary group $\mathfrak{U}(\mathfrak{l})$ acts on $A$ by automorphisms. It is easy to get such a multiplicative module out of a Fredholm module $(\mathfrak{h}, D)$ by the CAR construction, and the possibility of replacing the CAR algebra by the algebras of $\mathrm{V}$. Jones and Ocneanu served as one motivation for the search of an action of $G L(\infty)$.

The second author was motivated by statistical mechanics, in particular by the transfer matrix method, and the problem of understanding critical phenomena and the continuum limit in classical models such as those of Potts and of Andrews et al.

The transfer matrix method allows us to reduce a two-dimensional classical or commutative statistical mechanical model to a one-dimensional quantum or non-

* Partially supported by the Science and Engineering Research Council 
commutative model. Subsequently, taking a continuum limit one obtains an Euclidean field theory, equipped with representations of the Virasoro algebra in the case of conformal invariant field theory. This framework is now well understood in the case of the nearest neighbour two dimensional Ising model [P, $\mathrm{AE}, \mathrm{EL}, \mathrm{A}, \mathrm{CE}, \mathrm{K}]$. This paper is the first part of an investigation into understanding critical phenomena in other classical models, such as those of Potts [B] and Andrews et al. [ABF], and representations of the Virasoro algebra at the level of some one dimensional quantum model (before the scaling limit takes place) set in the operator algebras of Temperley and Lieb [TL], Jones [J], and Ocneanu [O]. Such algebras can be regarded as deformations of the Clifford algebra. This investigation begins here with a study of embeddings of $U(1)$-current algebras in the one-dimensional quantum systems.

Consider the following two dimensional classical models on the square lattice $\mathbb{Z}^{2}$ :

(a) The Potts model [B]. At each site we have $q$-possible states, so that a configuration of the system is an element of $\{1,2, \ldots, q\}^{\mathbb{Z}^{2}}$. The Hamiltonian for the (standard) Potts model is $\mathscr{H}(\sigma)=-\sum_{\alpha, \beta} J \delta\left(\sigma_{\alpha}, \sigma_{\beta}\right)$, where the summation is over nearest neighbours.

(b) The Andrews-Baxter-Forrester model [ABF]. Again we have $\ell$ possible states at each site, but we only consider configurations $\sigma \in\{1,2, \ldots, \ell\}^{\mathbb{Z}^{2}}$ such that $\left|\sigma_{\alpha}-\sigma_{\beta}\right|=1$ for nearest neighbours $\alpha, \beta$. The Hamiltonian is obtained by considering interactions around faces.

In the algebraic approach, we reduce the problem set classically in $C(P)$, the continuous functions on a configuration space $P$ to a non-commutative $C^{*}$-algebra $A[\mathrm{P}, \mathrm{AE}, \mathrm{CE}, \mathrm{K}]$. The programme is, given a classical equilibrium state $\mu$ at inverse temperature $\beta$, to find a linear map $\phi_{\mu}$ on the $C^{*}$-algebra $A$, and a map $F \rightarrow F_{\beta}$ from local observables in $C(P)$ to $A$, such that a classical correlation function can be computed using a knowledge of the quantum system alone:

$$
\phi(F)=\phi_{\mu}\left(F_{\beta}\right) \text {. }
$$

One expects that the map $F \rightarrow F_{\beta}$ depends only on the inverse temperature $\beta$, and that positivity of $\phi_{\mu}$ is related to reflection positivity of $\mu[\mathrm{K}]$.

Take for example the one dimensional 2-state Potts model [B]. This can be described using the algebra $M_{q}$ of complex $q \times q$ matrices. The Hamiltonian for the Potts model with periodic boundary conditions is

$$
\mathscr{H}_{L}(\sigma)=-\sum_{i=-L}^{L} J \delta\left(\sigma_{i}, \sigma_{i+1}\right) .
$$

Then the partition function is

$$
Z_{L}=\sum_{\sigma} \exp -\beta \mathscr{H}(\sigma)=\sum_{\sigma} \exp \left(\beta J \delta\left(\sigma_{i}, \sigma_{i+1}\right)\right)=\operatorname{tr} T^{2 L+1}
$$

if $T_{\sigma, \sigma^{\prime}}=\exp \left(\beta J \delta\left(\sigma, \sigma^{\prime}\right)\right)$, a $q \times q$ matrix when $\sigma, \sigma^{\prime} \in\{1,2, \ldots, q\}$. Similarly

$$
\langle F\rangle_{\beta}^{L}=\sum F(\sigma) \exp -\beta \mathscr{H}(\sigma) / Z_{L}=\operatorname{tr} F_{\beta} T^{2 L+1} / \operatorname{tr} T^{2 L+1} .
$$

Consequently, letting $L \rightarrow \infty$, we have

$$
\langle F\rangle_{\beta}=\left\langle F_{\beta} \Omega, \Omega\right\rangle,
$$


where $\Omega=q^{-1 / 2}(1, \ldots, 1)$ is the largest eigenvector of the transfer matrix $T=\left(e^{K}-1\right) e^{K * f}$, if $\left(e^{K}-1\right)\left(e^{K *}-1\right)=q$, and $f$ is the rank one projection on $\Omega$.

One proceeds similarly in 2-dimensions by first taking a finite box $[-M, M]$ $\times[-L, L]$ and pushing each column $\{i\} \times[-L, L]$ onto one site $(i, 0)$ and picking up one copy of $M_{q}$ for each site $(i, 0)$ in the row $[-M, M] \times\{0\}$. Thus

$$
Z_{L M}=\operatorname{tr} T^{2 L+1},\langle F\rangle_{\beta}^{L M}=\operatorname{tr} F_{\beta}^{M} T^{2 L+1} / \operatorname{tr} T^{2 L+1},
$$

where now $T_{M}, F_{\beta}^{M} \in \underset{-M}{\otimes} M_{q}$, and one can proceed to take the thermodynamic limit as before. The transfer matrix $T$ can be expressed, up to a scalar, as $T=V^{1 / 2} W V^{1 / 2}$. Here $V$ comes from vertical interactions so that

and

$$
V=\exp K_{1}^{*} \sum_{i} e_{2 i}
$$

$$
\underset{2 i}{e_{2 i}=1 \otimes \ldots \otimes f \otimes 1 \ldots \otimes 1} \text { position } .
$$

We have taken different interaction constants $J_{1}, J_{2}$ in the vertical and horizontal directions with $K_{i}=\beta J_{i}$. Then $W$ arising from horizontal interactions is diagonal, and

$$
W=\exp K_{2} \sum_{i} e_{2 i+1}, e_{2 i+1}=1 \otimes \ldots \underset{i, i+1}{\otimes} g \otimes 1 \ldots \otimes 1,
$$

where $g=\sum_{j=1}^{q} g_{j} \otimes g_{j} \in M_{q} \otimes M_{q}$, and $g_{j}$ is the rank one projection on $\Omega_{j}=\left(\delta_{i j}\right)_{i=1}^{q}$. The family $\left\{e_{i}\right\}_{-\infty}^{\infty}$ of projections arising from the Potts model transfer matrix satisfy the relations $[\mathrm{J}, \mathrm{B}]$ :

$$
\begin{gathered}
e_{i} e_{j}=e_{j} e_{i},|i-j| \geqq 2, \\
e_{i} e_{i \pm 1} e_{i}=\tau e_{i},
\end{gathered}
$$

where $\tau^{-1}=q$. Similarly, one can describe the transfer matrix of the ABF model (at least at criticality) with the aid of projections satisfying similar relations with $\tau^{-1}=4 \cos ^{2} \pi /(\ell+1)$, [KAW, P]. We note from Jones' classification of index of subfactors $[\mathrm{J}]$, that an infinite family $\left\{e_{i}\right\}$ of projections exists satisfying (1.9) and (1.10) if and only if $\tau^{-1} \in[4, \infty) \cup\left\{4 \cos ^{2} \pi /(\ell+1): \ell=3,4, \ldots\right\}$. Observe that $2=4 \cos ^{2}(\pi / 4), 3=4 \cos ^{2}(\pi / 6)$. One can indeed generalise the ABF models [P] by taking any finite connected graph $\Gamma$, with vertices $\Gamma^{(0)}$ and edges $\Gamma^{(1)}$. Then consider the space of configurations $\sigma \in\left(\Gamma^{(0)}\right)^{\mathbb{Z}^{2}}$ subject to the constraint that $\left(\sigma_{\alpha}, \sigma_{\beta}\right) \in \Gamma^{(1)}$ for all pairs $\alpha, \beta$ of nearest neighbours in $\mathbb{Z}^{2}$. Then in this model, one gets a representation of the Jones' relations where $\tau^{-1}=\alpha^{2}$, and $\alpha$ is the largest eigenvalue of a certain incidence matrix associated with the graph $\Gamma$.

From a lattice model, one gets a field theory by taking a continuum limit; letting the lattice spacing $\varepsilon \rightarrow 0$, whilst simultaneously letting the temperature approach the critical temperature, in such a way that $\varepsilon \cdot$ (correlation length) is constant. 
This is well understood in the Ising model [SMJ, PT1, PT2, SO'C]. Belavin et al. [BPZ] suggested using conformal invariance to understand scale invariance of special critical points. Conformal invariance is understood via representations of the Virasoro algebras. A knowledge of which representations can appear tells us something about the field theory and the nature of the critical points. The Virasoro algebra is the unique central extension of the Witt algebra of vector fields on $S^{1}$, generated by $L_{m}, m \in \mathbb{Z}$ and a central element $c$ with

$$
\left[L_{m}, L_{n}\right]=(m-n) L_{m+n}+\frac{c}{12} m\left(m^{2}-I\right) \delta_{m,-n} .
$$

Unitarity of the field theory, (coming from reflection positivity of the statistical mechanical model) means $L_{n}^{*}=L_{-n}$. From physical considerations, one is also interested in highest weight representations, where the energy of $L_{0}$ is bounded below. There is a vector $|h\rangle$, the highest weight vector, a scalar $h$ such that $L_{0}|h\rangle$ $=h|h\rangle, L_{n}|h\rangle=0, n>0$. In an irreducible representation, $c$ is a scalar, and so an irreducible highest weight unitary representation is determined by a pair $(c, h)$ of real numbers. It is known that such representations exist if and only if either [FQS, GKO]:

$$
\begin{gathered}
c \geqq 1, \quad h \geqq 0, \\
c=1-6 / m(m+1), \quad h=h_{p, q}(c)=\left[((m+1) p-m q)^{2}-1\right] / 4 m(m+1), \\
m=2,3,4, \ldots, p=1,2, \ldots, m-1, \quad q=1,2, \ldots, p .
\end{gathered}
$$

The question then arises of which representations correspond to certain statistical mechanical models. The possible values of $h$ are related to the scaling dimensions or critical exponents. In this way, comparing with known exponents, one can match [FQS, H] (1.13) $m=3$ with the Ising model, $m=4$ with the tricritical Ising model, $m=5$ with the 3 -state Potts model, and $m \geqq 5$ with the ABF models. On the other hand we have noted how the Potts model and ABF models are related to the Jones' classification via the transfer matrix method. It is thus natural to look for a direct link between the FQS classification and that of Jones. In this present paper we construct $U(1)$-currents directly from the Jones algebra, from which, using the Sugawara formula one can construct representations of the Virasoro algebra with central charge $c=1$.

The operator algebras of Jones and Ocneanu provide a natural setting for deforming the Fermi-Dirac quantization of the classical fields on $S^{1}$. The Jones algebra $A(\tau)$ is the $C^{*}$-algebra generated by projections $\left\{e_{i}\right\}_{i \in \mathbb{Z}}$ satisfying relations (1.9) and (1.10). The Ocneanu algebra (see Sect. 3) $A(\Gamma)$ is a slightly larger algebra associated to a connected graph $\Gamma$ such that the largest eigenvalue of its associated incidence matrix is $\tau^{-1 / 2}$. In general $A(\Gamma)$ is generated by $A(\tau)$ and a finite dimensional $C^{*}$-algebra. In Sect. 2 we show how to embed a copy of an observable algebra in an operator algebra, simply by constructing certain representations of the Lie algebra $g l(\infty)$ from operators satisfying infinitesimal versions of the Jones' relations. This allows us in Sects. 3 and 4 to embed the observable algebra in the algebras of Jones and Ocneanu, with the Ocneanu algebra permitting a rich choice of embeddings. The Hamiltonian $L_{0}=\sum n e_{2 n}$ generates time evolution, and has a unique KMS state $\omega_{\beta}$ for each temperature $\beta$ (if $\tau^{-1}<4$ ), and a canonical ground 
state $\omega_{\beta}$ (Sect. 5). The restriction of $\omega_{\beta}$ to any observable algebra constructed in Sect. 4 is quasi-free. In the state $\omega_{\infty}$ we can construct (Sect. 6) $U(1)$-currents $T_{n}$ satisfying $\left[T_{m}, T_{n}\right]=n \delta_{m,-n}$, to which one can apply the Sugawara formula.

What we have achieved is to construct representations of the Virasoro algebra in the setting of the algebras of V. Jones and Ocneanu, with central charge $c=1$. It remains to construct representations of the Virasoro algebra with central charge $c=1-6 / m(m+1)$ in the setting of the algebras associated with index $\tau^{-1}$ $=4 \cos ^{2} \pi /(m+1)$. However, we have strong evidence from studying the characters of the discrete series of representations of the Virasoro algebra that this can be done. It also remains to understand how the families of $U(1)$-currents which we have constructed, associated to a single graph $\Gamma$, fit together.

\section{Representations of $g l(\infty)$ and of the Observable Algebra}

If $I$ is a countable set, let $g l(I)$ denote the Lie algebra of $|I| \times|I|$ matrices over $\mathbb{C}$, with only finitely many non-zero entries, generated by the elementary matrices $\left\{E_{i j}: i, j \in I\right\}$. The first lemma shows the equivalence between those representations of $g l(I)$, where $E_{i i}$ are projections, with representations of the observable algebra over the Hilbert space $\ell^{2}(I)$.

Lemma 2.1. Let I be a countable set, $\mathscr{B}$ a unital $C^{*}$-algebra, and for each $i, j \in I$, let $e_{i j}$ be elements of $\mathscr{B}$. Then the following pair of conditions are equivalent:
(a) $e_{i i}=e_{i i}^{2}$
(b) $e_{i j}^{*}=e_{j i}$,
(c) $\left[e_{i j}, e_{j l}\right]=e_{i i}-e_{j j}$,
(d) $\left[e_{i j}, e_{j k}\right]=e_{i k}$ if $j \neq k$,
(e) $\left[e_{i j}, e_{k l}\right]=0$ if $j \neq k, l \neq i$.

(2.2) There is an unique unital *-homomorphism $\pi$ from the observable algebra over $\ell^{2}(I)$ into $\mathscr{B}$ such that $\pi\left(a_{i}^{*} a_{j}\right)=e_{i j}$.

Proof. (2.2) $\Rightarrow(2.1)$ is clear as $E_{i j} \rightarrow a_{i}^{*} a_{j}$ extends to a Lie algebra representation of $g l(I)$.

$(2.1) \Rightarrow(2.2)$. Suppose $I$ is ordered, say an interval in $\mathbb{Z}$.

Let $f_{i}=e_{i i}, u_{i}=e_{i, i+1}$. From (2.1)(d) we have $\left.f_{i} u_{i}-u_{i} f_{i}=u_{i},{ }^{*}\right)$. Multiplying (*) on the left by $f_{i}$, we see $f_{i} u f_{i}=0$, and then multiplying $\left(^{*}\right)$ on the right by $f_{i}$ we see $u_{\imath} f_{i}=0$, and so $f_{i} u_{i}=u_{i}$. Similarly, $u_{i}^{*} f_{i+1}=0, u_{i}^{*} f_{i}=u_{i}^{*}$. Hence from (2.1)(c), $\left[u_{i}, u_{i}^{*}\right]$ $=f_{i}-f_{i+1}$, we see $u_{i}^{*} u_{i}=f_{i+1}\left(1-f_{i}\right), u_{i} u_{i}^{*}=f_{i}\left(1-f_{i+1}\right)$. Then Lemma 2.1 will follow from:

Lemma 2.2. Let $I$ be a countable set, say an interval in $\mathbb{Z}$. For each $i \in I$, let $f_{i}, u_{i}$ be elements of a unital $C^{*}$-algebra $\mathscr{B}$. Then the following three sets of conditions are equivalent:

(2.3) Each $f_{i}$ is a projection and the map $E_{i i} \rightarrow f_{i}, E_{i, i+1} \rightarrow u_{i}$ extends to a Lie algebra homomorphism from $\mathrm{gl}(I)$ into $\mathscr{B}$.

(2.4) There is an unique unital *-homomorphism $\pi$ from the observable algebra over $I^{2}(I)$ into $\mathscr{B}$ such that $\pi\left(a_{i}^{*} a_{i}\right)=f_{i}, \pi\left(a_{i}^{*} a_{i+1}\right)=u_{i}$. 
(a) $f_{i}$ is a projection,

(b) $\left[f_{i}, f_{j}\right]=0$,

(c) $\left[u_{i}, f_{j}\right]=0$ if $j \neq i, i+1$,

(d) $\left[u_{i}, u_{j}^{\#}\right]=0$ if $|i-j| \geqq 2$,

(e) $u_{i}^{*} u_{i}=f_{i+1}\left(1-f_{i}\right), u_{i} u_{i}^{*}=f_{i}\left(1-f_{i+1}\right)$.

Proof. (2.5) $\Rightarrow(2.4)$. Let $K=\{-1,1\}^{I}$ with the equivalence relation $\sim$ on $K$ given by $x \sim y$ if $x=(x(i)), y=(y(i)) \in K, x(i)=y(i)$ except for finitely many coordinates, and $\sum_{i \in I}(x(i)-y(i))=0$. We identify the gauge invariant CAR algebra on $\ell^{2}(I)$ with the $C^{*}$-algebra $C^{*}(G)$ of the groupoid of the equivalence relation $G$ of $\sim[\mathrm{R}]$. In particular $C^{*}(G)$ contains $C(K)$, and so $g_{i}$, the projection given by the characteristic function of $\{x \in K: x(i)=1\}$. Let $v_{i}$ be the partial isometry with initial support $g_{i+1}\left(1-g_{i}\right)$ and final support $g_{i}\left(1-g_{i+1}\right)$, which replaces $x(i)=-1, x(i+1)=1$ with $x(i)=1, x(i+1)=-1$, which does not affect $\sum x(i)$ and so $v_{i}$ belongs to $C^{*}(G)$. The $v_{i}$ generate the equivalence relation, since given any sequence $(-1,1,-1, \ldots, 1)$ of \pm 1 , one can always put the -1 's on the left by replacing any pair $1,-1$ by $-1,1$. Thus to show that $\pi: g_{i}, v_{i} \rightarrow f_{i}, u_{i}$ extends to a homomorphism between the $C^{*}$-algebras $C^{*}(G)$ and $\mathscr{B}$, one just has to check that it gives a covariant representation of a groupoid. It is clear that $\pi: g_{i} \rightarrow f_{i}$ extends to a homomorphism of $C(K)$ onto $C^{*}\left(f_{i}\right)$. To show that $U: v_{i} \rightarrow u_{i}$ extends to a representation of the inverse semigroup $\mathscr{G}$ of partial isometries on $K$, one notes that $\mathscr{G}$ is the universal inverse semigroup of partial isometries on generators $\left\{v_{i}, v_{i}^{*}, f_{i}, 1-f_{i}\right\}$ subject to the relations $2(\mathrm{a})-(\mathrm{e})$, which imply the infinitesimal Jones or braid relations:

$$
v_{i} v_{i \pm 1} v_{i}=0 \quad v_{i}^{2}=0
$$

together with $v_{i} v_{i \pm 1}^{*}=0=v_{i \pm 1}^{*} v_{i}$. To see that $\mathscr{G}$ is universal one merely observes that any non-zero word in $\left\{v_{i}, v_{i}^{*}, f_{i}, 1-f_{i}\right\}$ can be uniquely expressed as

where

$$
f(\varepsilon) v^{\#}\left(J_{1}\right) \ldots v^{\#}\left(J_{p}\right)
$$

(a) $f(\varepsilon)=\prod_{i} f\left(\varepsilon_{i}\right), f\left(\varepsilon_{i}\right)=1, f_{i}$ or $1-f_{i}$, and $\operatorname{supp} \varepsilon=\left\{i: f_{i} \neq 1\right\}$ is finite.

(b) $J_{i}=\left\{k_{i}, k_{i}+1, \ldots, j_{i}\right\} j_{i} \geqq k_{i}, j_{i+1}>j_{i}, k_{i+1}>k_{i}, v^{\#}\left(J_{i}\right)=v_{k_{\mathrm{\imath}}}^{\#} \ldots v_{j_{\mathrm{\imath}}}^{\#}$. We say $v^{*}\left(J_{i}\right)$ is starred, $v\left(J_{i}\right)$ is unstarred.

(c) If $J_{i}^{0}=\left\{k_{i}, \ldots, j_{i}+1\right\}$, and one of $\left\{v^{\#}\left(J_{i}\right), v^{\#}\left(J_{i+1}\right)\right\}$ is starred and one is unstarred, then $J_{i}^{0} \cap J_{i+1}=\emptyset$.

(d) $\operatorname{supp}(\varepsilon) \cap \bigcup_{i=1}^{p} J_{i}^{0}=\emptyset$.

We can then deduce that $(\pi, U)$ gives a covariant representation of the groupoid $\mathscr{G}$ on $K$ and so gives the required embedding of the observable algebra on $\ell^{2}(I)$ in $\mathscr{B}$.

Remark 2.3. The relations (2.6) give rise to Quantum Yang-Baxter equation in that $R_{i}(s)=\exp \left(s v_{i}\right)$ satisfy:

$$
\begin{gathered}
R_{i}(s) R_{i+1}\left(s+s^{\prime}\right) R_{i}\left(s^{\prime}\right)=R_{i+1}\left(s^{\prime}\right) R_{i}\left(s+s^{\prime}\right) R_{i+1}(s), \\
R_{i}(s) R_{j}\left(s^{\prime}\right)=R_{j}\left(s^{\prime}\right) R_{i}(s) \quad|i-j| \neq 1
\end{gathered}
$$




\section{Local Structure}

We start with a $C^{*}$-algebra $A$ with the following type of local structure over $\mathbb{Z}$. To every subset $\Lambda \subset \mathbb{Z}$ is associated a $C^{*}$-subalgebra $A(\Lambda) \subset A$ in such a way that

$$
\begin{gathered}
\text { if } \Lambda \subset \Lambda^{\prime} \text {, then } A(\Lambda) \subset A\left(\Lambda^{\prime}\right) \text {, } \\
\text { if } d\left(\Lambda, \Lambda^{\prime}\right) \geqq 1 \text {, then } A(\Lambda) \text { and } A\left(\Lambda^{\prime}\right) \text { commute. }
\end{gathered}
$$

Here $d\left(\Lambda, \Lambda^{\prime}\right)=\min \left\{|i-j|: i \in \Lambda, j \in \Lambda^{\prime}\right\}$. We are also given an automorphism $\sigma$ of $A$ such that

$$
\sigma(A(\Lambda))=A(\Lambda+2), \text { for any } \Lambda \in \mathbb{Z} .
$$

Example 3.1. The Jones Algebra. If $\tau^{-1} \in[4, \infty) \cup\left\{4 \cos ^{2} \pi / m: m=3,4, \ldots\right\}$, let $A=A_{\tau}=A(\tau)$ be the $C^{*}$-algebra generated by projections $e_{n}, n \in \mathbb{Z}$ satisfying the $\mathrm{V}$. Jones' relations

$$
\begin{gathered}
e_{n} e_{m}=e_{m} e_{n}, \quad|m-n| \geqq \tau, \\
e_{n} e_{n \pm 1} e_{n}=\tau e_{n} .
\end{gathered}
$$

For each subset $\Lambda \subset \mathbb{Z}$, let $A(\Lambda)$ be the $C^{*}$-subalgebra generated by $e_{n}, n \in \Lambda$. Let $\sigma$ be the shift of $2, \sigma\left(e_{n}\right)=e_{n+2}$, so that conditions (3.1)-(3.3) are obviously satisfed.

There is an unique positive trace $\operatorname{Tr}$ on $A(\tau)$ called the Markov trace, such that

$$
\begin{gathered}
\operatorname{Tr}\left(x e_{m}\right)=\tau \operatorname{Tr}(x) \quad x \in A(-\infty, m-1], \\
\operatorname{Tr}\left(e_{m}\right)=\tau, \quad \operatorname{Tr}(1)=1 .
\end{gathered}
$$

In particular, for $\tau^{-1}=\frac{1}{2}=4 \cos ^{2} \pi / 4, A\left(\frac{1}{2}\right)$ can be identified with the even part of the Clifford algebra over $l_{\mathbb{R}}^{2}(\mathbb{Z})$. More precisely let $\left\{\gamma_{n}: n \in \mathbb{Z}\right\}$ satisfy the Clifford relations $\gamma_{n}=\gamma_{n}^{*},\left\{\gamma_{n}, \gamma_{m}\right\}=2 \delta_{m n}$, so that $U_{n}=i \gamma_{n} \gamma_{n+1}$ are self adjoint unitaries in the Clifford algebra $A^{F}=C^{*}\left(\gamma_{n}: n \in \mathbb{Z}\right)$ satisfying the relations $U_{n} U_{m}=U_{m} U_{n}$, $|m-n| \geqq 2$, and $U_{n} U_{n+1}=-U_{n+1} U_{n}$. Hence $e_{n}=\left(1+U_{n}\right) / 2=\left(1+i \gamma_{n} \gamma_{n+1}\right) / 2$ satisfy the V. Jones relations (3.4) and (3.5), so that $A\left(\frac{1}{2}\right)$ can be identified with the even part of the Clifford algebra, namely $A_{+}^{F}=C^{*}\left(\gamma_{i} \gamma_{j}: i, j \in \mathbb{Z}\right)$. (The algebra $A^{F}$ is graded so that $\gamma_{n}$ is odd.) Going from this real or Clifford picture to a CAR picture, one takes a complex structure on $l_{\mathbb{R}}^{2}(\mathbb{Z})$ with real basis $\eta_{n}$ such that $i \eta_{2 n}=\eta_{2 n+1}$, $i \eta_{2 n+1}=-\eta_{2 n}$. Then the creation operators $a_{n}=\left(\gamma_{2 n}+i \gamma_{2 n+1}\right) / 2$, are odd, and satisfy the canonical commutation relations $\left\{a_{n}, a_{m}\right\}=0,\left\{a_{n}, a_{m}^{*}\right\}=\delta_{n m}$. In this language we identify:

$$
\begin{gathered}
\mathcal{c}_{2 n}=a_{n}^{*} a_{n}, \\
e_{2 n+1}=\left[1+\left(a_{n}-a_{n}^{*}\right)\left(a_{n+1}+a_{n+1}^{*}\right)\right] / 2 .
\end{gathered}
$$

Then $a_{n}^{*} a_{n}=e_{2 n}, a_{n}^{*} a_{n+1}=e_{2 n}\left(1-2 e_{2 n+1}\right) e_{2 n+2}$ defines a Lie algebra homomorphism of $g l(\infty)$ into $A\left(\frac{1}{2}\right)$.

Example 3.2. The Ocneanu Algebra. Let $\Gamma$ be a graph, with base point ${ }^{*}, \Gamma^{(0)}$ the set of vertices, $\Gamma^{(1)}$ the set of edges, so that $\Gamma^{(1)}$ consists of certain two element subsets of $\Gamma^{(0)}$. We say that $\gamma \in \Gamma^{(0)}$ is even (respectively odd) if it can be joined to * 
by an even (respectively odd) number of edges. We let $\Gamma_{+}^{(0)}$ (respectively $\Gamma_{-}^{(0)}$ ) denote the even (respectively odd) vertices. Let $K$ be the Cantor set of all sequences $\left(x_{v}\right)_{v \in \mathbb{Z}}$ with

$$
\begin{gathered}
\forall v,\left(x_{v}, x_{v+1}\right) \in \Gamma^{(1)}, \\
x_{0} \text { is even. }
\end{gathered}
$$

On $K$ consider the equivalence relation $\sim$ with countable orbits given by $\left(x_{v}\right) \sim\left(y_{v}\right)$ if and only if $x_{v}=y_{v}$, except in some finite set of $v^{\prime}$ 's. Let $A=A^{\Gamma}=A(\Gamma)$ be the corresponding $C^{*}$-algebra. For each (finite) subset $\Lambda \subset \mathbb{Z}$, let $A(\Lambda)=A^{\Gamma}(\Lambda)$ be the $C^{*}$-subalgebra generated by the following partial isometries $f_{\gamma \gamma^{\prime}}$ : Both $\gamma$ and $\gamma^{\prime}$ are elements of

$$
\begin{aligned}
\mathscr{G}^{A}= & \left\{\gamma \in\left(\Gamma^{(0)}\right)^{\Lambda^{\prime}}:\left(\gamma_{i}, \gamma_{i+1}\right) \in \Gamma^{(1)}, \gamma_{2 m} \in \Gamma_{+}^{(0)}, \gamma_{2 n+1} \in \Gamma_{-}^{(0)},\right. \\
& \text { if } \left.i, i+1,2 m, 2 n+1 \in \Lambda^{\prime}\right\},
\end{aligned}
$$

where $\Lambda^{\prime}=\{i: d(i, \Lambda) \leqq 1\}$, and $\gamma(j)=\gamma^{\prime}(j)$ if $j \notin \Lambda$. The partial isometry $f_{\gamma \cdot \gamma^{\prime}}$ has as initial domain the cylinder set $Z_{\gamma}=\left\{\left(x_{v}\right): x_{i}=y_{i}, i \in \Lambda^{\prime}\right\}$, and it replaces any $\operatorname{such}\left(x_{v}\right)$ in $Z_{\gamma}$, by $\left(y_{v}\right)$ in the cylinder set $Z_{\gamma^{\prime}}$ where $y_{v}=x_{v}, v \notin \Lambda, y_{v}=\gamma_{v}^{\prime}, v \in \Lambda$. The shift by 2 on $K$ induces an automorphism $\sigma$ of $A(\Gamma)$ satisfying (3.1)-(3.3).

Associated to the graph $\Gamma$ is a symmetric $0-1$ matrix $A$, indexed by the vertices $\Gamma^{(0)}$, with entries $A_{\varrho \eta}$ equal to 0 or 1 , according as $(\varrho, \eta) \in \Gamma^{(1)}$ or $(\varrho, \eta) \notin \Gamma^{(1)}$. We let $t(\eta)=\left\{\varrho \in \Gamma^{(0)}:(\varrho, \eta) \in \Gamma^{(1)}\right\}$. If the graph is connected, then there exists an unique eigenvector $\left(v_{\varrho}\right)_{\varrho \in \Gamma^{(0)}}$ with strictly positive entries, and $\alpha \in(0,1]$ such that

$$
v_{\eta}=\sum_{\varrho \in t(\eta)} \alpha v_{\varrho}, \quad \sum_{\eta \text { even }} v_{\eta}^{2}=1
$$

Then $X(\eta)=\left(\left(\alpha v_{\varrho} / v_{\eta}\right)^{1 / 2}\right)_{\varrho \in \Gamma^{(0)}}$ defines a unit vector in $\ell^{2}(t(\eta))$. If $k \leqq \ell \in \mathbb{Z}, \delta, \beta \in \Gamma^{(0)}$, let $\mathscr{G}_{\delta, \beta}^{[k, \ell]}=\left\{\gamma \in \mathscr{G}^{[k, \ell]}: \gamma(k-1)=\delta, \gamma(\ell+1)=\beta\right\}$. Then for each $n \in \mathbb{Z}$, let

$$
e_{n}=\sum_{\eta} X(\eta)(\varrho) X(\eta)\left(\varrho^{\prime}\right) f_{\gamma, \gamma^{\prime}}
$$

where the summation is over all $\eta \in \Gamma^{(0)}$ if $n$ is even or all $\eta \in \Gamma_{+}^{(0)}$ if $n$ is odd, $\gamma, \gamma^{\prime} \in \mathscr{G}_{\eta, \eta}^{\{n\}}$, and $\gamma(n)=\varrho, \gamma^{\prime}(n)=\varrho^{\prime}$. Then $e_{n}$ is a projection, being identified with a sum (over odd or even vertices depending on whether $n$ is even or odd) of the rank one projection on $X(\eta)$ in $\operatorname{End}\left(\ell^{2}(t(\eta))\right)$. The family $e_{n}$, satisfy the Jones' relations (3.3) and (3.4) for $\tau=\alpha^{2}$, so that $A\left(\alpha^{2}\right) \subset A(\Gamma)$.

We define a trace $\mathrm{Tr}$, called the Markov trace, on $A(\Gamma)$ to be the unique state on $A(\Gamma)$ such that

$$
\begin{gathered}
\operatorname{Tr} f_{\gamma, \gamma^{\prime}}=0 \quad \text { if } \gamma \neq \gamma^{\prime}, \\
\operatorname{Tr} f_{\gamma, \gamma}=\alpha^{\ell-k+2} v_{\delta} v_{\beta}, \quad \text { if } \gamma \in \mathscr{G}_{\delta, \beta}^{[k, \ell]} .
\end{gathered}
$$

Then

$$
\begin{gathered}
\operatorname{Tr}\left(x e_{m}\right)=\alpha^{2} \operatorname{Tr}(x), x \in A(-\infty, m-1], \\
\operatorname{Tr}\left(e_{m}\right)=\alpha^{2}, \operatorname{Tr}(1)=1 .
\end{gathered}
$$


Consequently $\operatorname{Tr} \mid A\left(\alpha^{2}\right)$ is the Markov trace on $A\left(\alpha^{2}\right)$. that

We emphasize that $A(\Gamma) \neq A\left(\alpha^{2}\right)$ in general, and we shall indeed see in Sect. 6

$$
A^{\alpha^{2}}\{0,1,2\}_{e_{0}\left(1-e_{2}\right)}=\mathbb{C} \neq A^{\Gamma}\{0,1,2\}_{e_{0}\left(1-e_{2}\right)} .
$$

However we claim that $A(\Gamma)$ is generated by $A\left(\alpha^{2}\right)$ and a finite dimensional algebra. Let $\varrho$ (respectively $\pi$ ) denote the left (respectively right) action of $A_{k}$ on $A_{k}$ so that $\varrho\left(A_{k}\right)^{\prime}=\pi\left(A_{k}\right)$. We first note that there is an action $\varrho$ of $A_{k+1}=A[0, k+1]$ on $A_{k}$, whose commutant is the (right) action of $A_{k-1}$ on $A_{k}$. The right action $\pi$ of $A_{k-1}$ on $A_{k}$ is given by

$$
\pi\left(f_{\gamma_{1}, \gamma_{1}^{\prime}}\right): f_{\gamma, \gamma^{\prime}} \rightarrow f_{\gamma, \gamma_{1}^{\prime} \varrho}
$$

if $\gamma^{\prime}=\gamma_{1}{ }^{\varrho}, \gamma_{1} \gamma^{\prime} \in \mathscr{G}^{[0, k]}, \gamma_{1}, \gamma_{1}^{\prime} \in \mathscr{G}^{[0, k-1]}, \varrho \in \mathscr{G}^{[k-1, k]}$, and $\pi\left(f_{\gamma_{1}, \gamma_{1}^{\prime}}\right) f_{\gamma \cdot \gamma^{\prime}}=0$ otherwise. To describe the commutant of the action of $\left\{f_{\gamma_{1}, \gamma_{1}^{\prime}}: \gamma_{1}^{\prime}(k-1)=\delta\right\}$ on $f_{\gamma, \gamma^{\prime}}$ we note that we have the freedom to change $\tilde{\gamma} \in[0, k+2]$, where $\tilde{\gamma} \mid[0, k]=\gamma, \tilde{\gamma}(k+1)=\delta$, which can be described as an action $\varrho$ of $A_{k+1}$ as follows:

$$
\varrho\left(f_{\tilde{\gamma}, \hat{\gamma}^{\prime}}\right): f_{\gamma, \gamma^{\prime}} \rightarrow v_{\eta_{1}}^{1 / 2} v_{\eta_{2}}^{-1 / 2} f_{\tilde{\gamma}_{0}, \gamma_{0}^{\prime}},
$$

if $\gamma, \gamma^{\prime} \in \mathscr{G}^{[0, k]}, \tilde{\gamma}, \tilde{\gamma}^{\prime} \in \mathscr{G}^{[0, k+1]}, \gamma_{0}^{\prime}\left|[0, k]=\gamma, \tilde{\gamma}_{0}=\tilde{\gamma}\right|[0, k], \gamma_{0}^{\prime}\left|[0, k-1]=\gamma^{\prime}\right|[0, k-1]$, $\gamma_{0}^{\prime}(k)=\tilde{\gamma}(k), \eta_{1}=\gamma(k)=\gamma^{\prime}(k), \eta_{2}=\tilde{\gamma}(k)$, and $\varrho\left(f_{\tilde{\gamma}, \tilde{\gamma}^{\prime}}\right) f_{\gamma, \gamma^{\prime}}=0$ otherwise. The factor $v_{\eta_{1}}^{1 / 2} v_{\eta_{2}}^{1 / 2}$ is inserted to make $\varrho$ a ${ }^{*}$-representation with respect to the inner product $\langle x, y\rangle=\operatorname{tr} y^{*} x$ on $A_{k-1}$. Then $\varrho\left(A_{k+1}\right)^{\prime}=\pi\left(A_{k}\right)$, and $\varrho$ is faithful if every vertex which is a right-hand end point of a path of length $k+1$, is also a right-hand end point of a path of length $k-1$ (with the same left-hand and points). We assume that this is the case. This will certainly be the case if the two restrictions of $A^{2}$ to even and odd vertices are irreducible matrices. Then $\varrho\left(e_{k+1}\right)=E_{k+1}$ is the orthogonal projection of $A_{k}$ on $A_{k-1}$.

Lemma 3.3. If $k$ is sufficiently large, $A_{k+1}$ is the $C^{*}$-algebra generated by $A_{k}$ and $e_{k+1}$.

Proof. We take $k$ sufficiently large that $\varrho$ is faithful. Let $x \in\left(\varrho\left(A_{k}\right), \varrho\left(e_{k+1}\right)\right)^{\prime}$ $\subset \varrho\left(A_{k}\right)^{\prime}=\pi\left(A_{k}\right)$. Then $x E_{k+1}=E_{k+1} x$ shows that $x=x E_{k+1}(I)=\left(E_{k+1} x\right) I$ $=E_{k+1}(x) \in A_{k-1}$. Hence $\quad\left(\varrho\left(A_{k}\right), \varrho\left(e_{k+1}\right)\right)^{\prime} C \pi\left(A_{k-1}\right), \quad$ and so $\varrho\left(A_{k+1}\right)$ $\supset\left(\varrho\left(A_{k}\right), \varrho\left(e_{k+1}\right)\right)^{\prime \prime} \supset \pi\left(A_{k-1}\right)^{\prime} \supset \varrho\left(A_{k+1}\right)$.

Corollary 3.4. The $C^{*}$-algebra $A(\Gamma)$ is generated by $A\left(\alpha^{2}\right)$ and a finite dimensional $C^{*}$-algebra.

\section{Embedding the Observable Algebra in Local Algebras}

Let $A$ be a $C^{*}$-algebra with local structure as in (3.1)-(3.3) of Sect. 3.

Lemma 4.1. Let $f_{0} \in A\{0\}$ be a projection and $u_{0} \in A\{0,1,2\}$ a partial isometry; with initial support $\sigma\left(f_{0}\right)\left(1-f_{0}\right)$ and final support $f_{0}\left(1-\sigma\left(f_{0}\right)\right)$. Then there exists an 
unique *homomorphism $j$ from the observable algebra $O$ over $\ell^{2}(\mathbb{Z})$ to $A$ such that

$$
\begin{gathered}
j \sigma_{\mathbb{d}}(x)=\sigma_{A}(x), x \in \mathcal{O}, \\
j\left(a_{0}^{*} a_{0}\right)=f_{0}, \\
j\left(a_{0}^{*} a_{1}\right)=u_{0} .
\end{gathered}
$$

Remark 4.2. Here $\sigma_{\mathscr{O}}$ is the automorphism of the observable algebra $\mathcal{O}$ which is the restriction of the automorphism $\sigma$ on the Fermion algebra given by $\sigma\left(\gamma_{n}\right)=\gamma_{n+2}$ or $\sigma\left(a_{i}\right)=a_{i+1}($ see Example 3.1).

Proof of Lemma 4.1. Let $f_{i}=\sigma^{i}\left(f_{0}\right) \in A\{i\}, u_{i}=\sigma^{i}\left(u_{0}\right) \in A\{2 i, 2 i+1,2 i+2\}$ for $i \in \mathbb{Z}$. Then $\left\{f_{i}, u_{i}\right\}$ satisfy conditions (2.5) of Lemma 2.2 .

Let us now examine Lemma 4.1 in the context of the Jones and Ocneanu algebras.

Lemma 4.2. Let $A_{\tau}$ be the $V$. Jones algebra as in Sect. 3.

(4.4) In $A\{0,1,2\}$ the projections $e_{0}\left(1-e_{2}\right)$ and $\left(1-e_{0}\right) e_{2}$ are equivalent.

(4.5) The reduced algebra of $A\{0,1,2\}$ by $e_{0}\left(1-e_{2}\right)$ is one-dimensional.

Proof. (4.4). If $u=\tau^{-1} e_{0}\left(1-e_{2}\right) e_{1}\left(1-e_{0}\right) e_{2}=e_{0}\left(1-\tau^{-1} e_{1}\right) e_{2} \in A\{0,1,2\}$, [cf. (Example 3.1)] then

$$
u^{*} u=\left(1-e_{0}\right) e_{2}, u u^{*}=e_{2}\left(1-e_{0}\right) .
$$

(4.5) is clear from (4.4), $A\{0,1,2\}=C^{*}\left(e_{0}, e_{1}, e_{2}\right)$ and the Jones' relations.

Theorem 4.3. (a) For $\tau^{-1} \in[4, \infty) \cup\left\{4 \cos ^{2} \pi / m\right\}$, let $A=A(\tau)$ the $V$. Jones algebra. Then for each $t \in \mathbb{T}$, there exists an unique *-endomorphism $j_{t}$ of the observable algebra 0 into $A(\tau)$ such that

$$
\begin{gathered}
j_{t} \sigma_{\mathcal{O}}(x)=\sigma_{A} j_{t}(x), \quad x \in \mathcal{O}, \\
j_{t}\left(a_{i}^{*} a_{i}\right)=e_{2 i}, \quad i \in \mathbb{Z}, \\
j_{t}\left(a_{i}^{*} a_{i+1}\right)=\sigma_{A}^{i}(t) e_{2 i}\left(1-\tau^{-1} e_{2 i+1}\right) e_{2 i+2}, \quad i \in \mathbb{Z} .
\end{gathered}
$$

(b) For $\Gamma$ a connected graph, let $A=A(\Gamma)$ be the Ocneanu algebra, and $G^{\Gamma}$ be the unitary group of the reduced algebra of $A^{\Gamma}\{0,1,2\}$ by $e_{0}\left(1-e_{2}\right)$. Then for each $t \in G^{\Gamma}$, there exists an unique *-endomorphism $j_{t}$ of the observable algebra $\mathcal{O}$ into $A(\Gamma)$ satisfying (4.6)-(4.8).

Proof. Using Lemmas 2.2 and 4.2, it only remains to show that the embeddings are faithful. This can be shown by computing the Markov trace on the projection $f(\varepsilon)$ (in the notation of the proof of Lemma 2.2) in the centre $C\left(( \pm 1)^{\mathbb{Z}}\right)$ of $\mathcal{O}$, and observing that this is non-zero.

It only remains to compute the group $G^{T}$.

Proposition 4.4. For $\Gamma$ a connected graph, let $A=A(\Gamma)$ be the Ocneanu algebra.

Any function of $x_{v}, v \in \Lambda^{\prime} \backslash \Lambda=\partial \Lambda$ lies in the centre of $A(\Lambda)$. 
(4.10) Let $q_{\eta}^{(n)}$ be the function $q_{\eta}^{(n)}\left(\left(x_{v}\right)\right)=0$ if $x_{n} \neq \eta$, and $q_{\eta}^{(n)}\left(\left(x_{v}\right)\right)=1$ if $x_{n}=\eta$. Then $q_{\eta}^{(-1)} e_{0} q_{\eta^{\prime}}^{(1)}=0$ if $\eta \neq \eta^{\prime}$.

(4.11) Let $P^{(k)}\left(\eta, \eta^{\prime}\right)$ denote the paths of length $k$ from $\eta$ to $\eta^{\prime}$. Then if $\eta \neq \eta^{\prime}$, $q_{\eta}^{(-1)} e_{0} q_{\eta}^{(1)}\left(1-e_{2}\right) q_{\eta^{\prime}}^{(3)}$ is a projection of dimension \# $P^{(2)}\left(\eta, \eta^{\prime}\right)$ in $A\{0,1,2\}$. (Here \# $(\emptyset)=0$.)

(4.12) $q_{\eta}^{(-1)} e_{0} q_{\eta}^{(1)}\left(1-e_{2}\right) q_{\eta}^{(3)}$ is a projection of dimension \#t( $\left.\eta\right)-1$ in $A\{0,1,2\}$.

Proof. (4.9): Any function of $x_{v}, v \in \partial \Lambda$ is a linear combination of $\left\{g_{\eta}^{(v)}: \eta\right\}$. Then $g_{\eta}^{(v)}$ $=\sum f_{\gamma, \gamma} \in A(\Lambda)$, where the summation is over all $\gamma \in \mathscr{G}^{A}$ such that $\gamma(\nu)=\eta$. Moreover $g_{\eta}^{(v)}$ is a unit for $\left\{f_{\gamma, \gamma^{\prime}}: \gamma, \gamma^{\prime} \in \mathscr{G}^{\Lambda}, \gamma\left|\partial \Lambda=\gamma^{\prime}\right| \partial \Lambda, \gamma(\nu)=\eta\right\}$ and annihilates $\left\{f_{\gamma, \gamma^{\prime}}: \gamma, \gamma^{\prime} \in \mathscr{G}^{\Lambda}, \quad \gamma\left|\partial \Lambda=\gamma^{\prime}\right| \partial \Lambda, \quad \gamma(v) \neq \eta\right\}$. Hence $g_{\eta}^{(v)}$ commutes with $A(\Lambda)$ $=\lim \left\{f_{\gamma, \gamma^{\prime}}: \gamma, \gamma^{\prime} \in \mathscr{G}^{\Lambda}, \gamma\left|\partial \Lambda=\gamma^{\prime}\right| \partial \Lambda\right\}$.

(4.10): We have

$$
A\{0\}=\underset{\eta, \eta^{\prime}}{\oplus} \operatorname{End} \ell^{2}\left(P^{2}\left(\eta, \eta^{\prime}\right)\right),
$$

where the summation is over odd $\eta, \eta^{\prime} \in \Gamma^{(0)}$. We have as in (4.9) that $q_{\eta}^{(-1)}$ (respectively $\left.q_{\eta}^{(1)}\right)$ is a unit for $\operatorname{End} \ell^{2}\left(P^{2}(\alpha, \beta)\right.$ ) if $\eta=\alpha$ (respectively $\eta=\beta$ ), and annihilates End $\ell^{2}\left(P^{2}(\alpha, \beta)\right)$ if $\eta \neq \alpha$ (respectively $\eta \neq \beta$ ). Hence $q_{\eta}^{(-1)}$ End $\ell^{2} P(\alpha, \alpha) q_{\eta^{\prime}}^{(1)}=0$ if $\eta \neq \eta^{\prime}$. Consequently $q_{\eta}^{(-1)} e_{0} q_{\eta^{\prime}}^{(1)}=0$ if $\eta \neq \eta^{\prime}$ as $e_{0} \in \underset{\alpha \text { odd }}{\oplus}$ End $\ell^{2}(P(\eta, \eta))$, [where we identify $P^{2}(\alpha, \alpha)$ with $t(\alpha)$.

(4.11): Take $\eta, \eta^{\prime}$ odd vertices in $\Gamma^{(0)}, \eta \neq \eta^{\prime}$. Then

$$
q_{\eta}^{(1)} q_{\eta^{\prime}}^{(3)}=\sum f_{\gamma^{\prime}, \gamma^{\prime}}
$$

where the summation is over all $\gamma^{\prime} \in \mathscr{G}_{\eta, \eta^{\prime}}^{\{2\}}$. Take $\gamma \in \mathscr{G}_{\eta}^{\{0\}}$, such that $q_{e_{0}}^{(-1)} e_{0} q_{\eta}^{(1)}$ being a rank one projection in End $\ell^{2}(t(\eta))$, there exists a unitary $u$ in End $\ell^{2}(t(\eta)) \in A\{0\}$ such that $q_{\eta}^{(-1)} e_{0} q_{\eta}^{(1)}=u f_{\gamma, \gamma} u^{*}$. Hence, since $\left[u, f_{\gamma^{\prime}, \gamma^{\prime}}\right]=0$ for each $\gamma^{\prime} \in \mathscr{G}_{\eta, \eta^{\prime}}^{(2)}$, we see that

$$
q_{\eta}^{(-1)} e_{0} q_{\eta}^{(1)}\left(1-e_{2}\right) q_{\eta^{\prime}}^{(3)}=q_{\eta}^{(-1)} e_{0} q_{\eta}^{(1)} q_{\eta^{\prime}}^{(3)}=u\left(\sum f_{\gamma \gamma^{\prime}, \gamma \gamma^{\prime}}\right) u^{*},
$$

where the summation is over all $\gamma^{\prime} \in \mathscr{G}_{\eta, \eta^{\prime}}^{\{2\}}$, and which is of dimension \# $P^{(2)}\left(\eta, \eta^{\prime}\right)$ in End $\left(\ell^{2}\left(P^{(2)}\left(\eta, \eta^{\prime}\right)\right)\right)$. (Here if $\gamma \in \mathscr{G}^{\Lambda_{1}}, \gamma^{\prime} \in \mathscr{G}^{\Lambda_{2}}$, with $\gamma=\gamma^{\prime}$ on $\Lambda_{1}^{\prime} \cap \Lambda_{2}^{\prime}$, we let $\gamma \gamma^{\prime}$ denote the obvious element of $\mathscr{G}^{\Lambda_{1} \cup \Lambda_{2}}$.)

(4.12): As in the proof of (4.11), we see that the dimension of $q_{\eta}^{(-1)} e_{0} q_{\eta}^{(1)}\left(1-e_{2}\right) q_{\eta}^{(3)}$ in End $\ell^{2}\left(P^{(4)}(\eta, \eta)\right)$ is the same as that of $q_{\eta}^{(1)}\left(1-e_{2}\right) q_{\eta}^{(3)}$ in End $\ell^{2}(t(\eta))$. Now $e_{2}$ is a rank one projection in End $\ell^{2}(t(\eta))$, and consequently its complement is of dimension \#t( $\eta)-1$.

Corollary 4.5. If $\Gamma$ is a connected graph, the reduction of the algebra $A^{\Gamma}\{0,1,2\}$ by $e_{0}\left(1-e_{2}\right)$ can be identified with

$$
\underset{\eta, \eta^{\prime} \text { odd }}{\oplus} M\left(N\left(\eta, \eta^{\prime}\right)\right)
$$

where $M(N)$ denotes the algebra of complex $N \times N$ matrices, and the summation is over odd vertices $\eta, \eta^{\prime}$ in $\Gamma^{(0)}$ with

$$
N\left(\eta, \eta^{\prime}\right)=\left\{\begin{array}{lll}
\# P^{(2)}\left(\eta, \eta^{\prime}\right) & \text { when } & \eta \neq \eta^{\prime} \\
\# t(\eta)-1 & \text { when } & \eta=\eta^{\prime}
\end{array}\right.
$$


and $\# \emptyset=0$. In particular, when $\Gamma$ is the graph of a simply laced Dynkin diagram (with base point * as indicated below) the group $G^{\Gamma}$ is as follows:

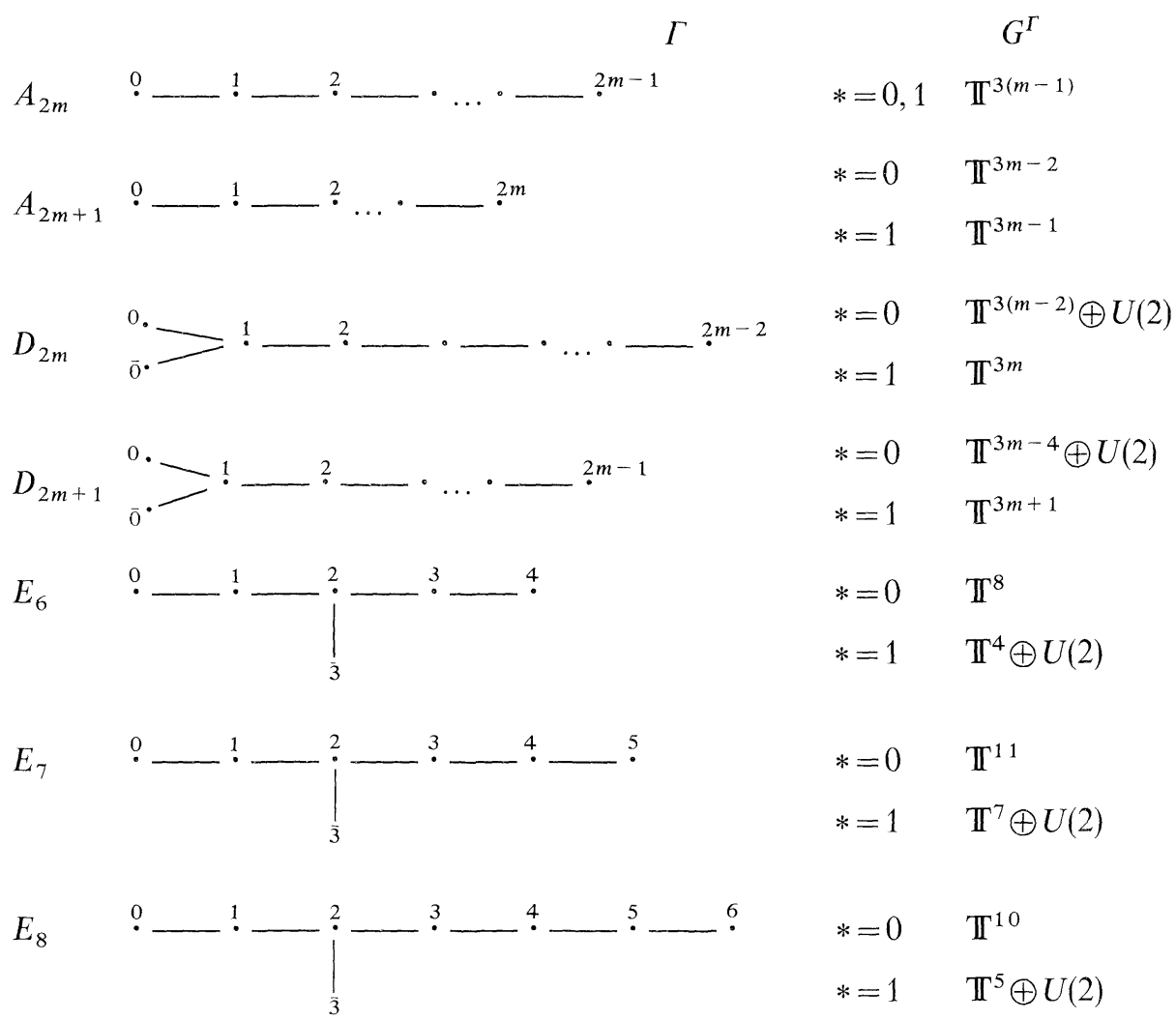

\section{The KMS States of the Hamiltonian $\sum n e_{2 n}$}

Let $\Gamma$ be a connected graph, with Jones index $\tau^{-1}$ as in Sect. 3. If $H_{m}=\sum_{|n| \leqq m} n e_{2 n}$, then $\lim _{m \rightarrow \infty} \operatorname{Ad}\left(e^{i H_{m} t}\right)$ defines a strongly continuous one parameter group $\sigma_{t}$ of *-automorphisms of $A(\Gamma)$, whose generator we denote by $L_{0}$. Clearly $\sigma_{t}$ leaves $A(\tau)$ and $j(\mathcal{O})$ invariant for any embedding $j$ of the observable algebra $\mathcal{C}$ in $A(\Gamma)$ as in Sect. 4 , and on such an observable algebra coincides with the time evolution given by the Dirac operator.

Theorem 5.1. Let $\Gamma$ be a connected graph with Jones' index $\tau^{-1}<4$.

(5.1) For each $\beta>0$, there exists an unique KMS state $\omega_{\beta}$ at inverse temperature $\beta$ for $L_{0}$. Moreover, the restriction $\omega_{\beta} \mid A(\tau)$ is also an unique KMS state for $L_{0} \mid A(\tau)$ at inverse temperature $\beta$.

(5.2) The states $\omega_{\beta}, \omega_{\beta} \mid A^{\tau}$ are factorial, and if $\tau^{-1} \neq 2$, of type $I I_{\infty}$.

(5.3) If $j$ is any embedding as in Sect. 5 of the observable algebra in $A(\Gamma)$, there exists a projection $E$ of norm one from $A(\Gamma)$ onto $j(\mathcal{O})$, such that $\omega_{\beta}=\psi_{\beta} \circ E$, where $\psi_{\beta}$ is the restriction of the quasi-free state $\omega_{A_{\beta}}$ to the observable algebra, and where 
$\omega_{A_{\beta}}$ is the unique KMS state on the Clifford algebra Cliff $\mathbb{C}_{\mathbb{C}}\left(\ell^{2}\left(S^{1}\right)_{\mathbb{R}}\right)$ for the Dirac Hamiltonian, with chemical potential $\beta^{-1} \log \left(\tau /(1-\tau)\right.$, namely $D_{\tau}=D$ $-\beta^{-1} \log \tau /(1-\tau)$. If $j=j_{t}$, for $t \in \mathbb{T} \subset G_{\Gamma}$, then $E$ takes $A(\tau)$ onto $j(\mathcal{O}) \subset A(\tau)$.

Proof. The Markov property of the unique trace $\operatorname{Tr}$ on $A(\Gamma)$ shows that $\omega(x)$ $=\lim _{m \rightarrow \infty} \operatorname{Tr}\left(e^{-\beta H_{m}} x\right) / \operatorname{Tr}\left(e^{-\beta H_{m}}\right)$ defines a state on $A(\Gamma)$, and which is KMS at inverse temperature $\beta$ for $\sigma_{t}$. Let $\phi$ be any KMS state for $\sigma_{t}$ at inverse temperature $\beta$, and $\phi_{m}=\phi / A_{m}, A_{m}=A^{T}[-2 m, 2 m]$. Then $\psi_{m}=\phi_{m}\left(e^{\beta H_{m}} \cdot\right) / \phi_{m}\left(e^{\beta H_{m}}\right)$ is a normalised positive trace on $A_{m}$. Let $\Delta_{m}$ denote the compact convex set of normalised positive traces on $A_{m}$, which can be identified with a subset of $\Delta_{m}=\left\{x \in \mathbb{R}^{p}: x_{i} \geqq 0, \sum x_{i}=1\right\}$, where $p=\left(\#\left(\Gamma^{(0)}\right)\right)^{2}$. Then by [E, Sects. 5-6], $\Delta_{m} \supset \Delta_{m+1}$, diameter $\Delta_{m} \rightarrow 0$, and $\bigcap_{m=1}^{\infty} \Delta_{m}$ can be identified with the unique normalised trace $\operatorname{Tr}$ on $A(\Gamma)$. Hence given $\varepsilon>0$, there exists $m_{0}$, such that for $m \geqq m_{0},(1-\varepsilon) \operatorname{Tr} \leqq \psi_{m} \leqq(1+\varepsilon) \operatorname{Tr}$. Hence for $m \geqq m_{0}$ :

$$
\begin{aligned}
\phi(x)-\operatorname{Tr}\left(e^{-\beta H_{m}} x\right) / \operatorname{Tr}\left(e^{-\beta H_{m}}\right) & =\psi_{m}\left(e^{-\beta H_{m}} x\right) / \psi_{m}\left(e^{-\beta H_{m}}\right)-\operatorname{Tr}\left(e^{-\beta H_{m}} x\right) / \operatorname{Tr}\left(e^{-\beta H_{m}}\right) \\
& \leqq\left((1+\varepsilon)(1-\varepsilon)^{-1}-1\right) \operatorname{Tr}\left(e^{-\beta H_{m}} x\right) / \operatorname{Tr}\left(e^{-\beta H_{m}}\right) .
\end{aligned}
$$

Thus $\phi(x)=\lim _{m \rightarrow \infty} \operatorname{Tr}\left(e^{-\beta H_{m}} x\right) / \operatorname{Tr}\left(e^{-\beta H_{m}}\right)=\omega(x)$, which shows uniqueness.

The state $\omega_{\beta} \circ j^{-1} \mid \mathcal{O}$ on the observable algebra $\mathcal{O}$ is independent of the choice of embedding $j$ of $\mathcal{O}$ in $A(\Gamma)$. In fact it is the restriction to $\mathcal{O}$ of the quasi-free state $\omega_{A_{\beta}}$, where $A_{\beta}=\tau e^{-\beta D} /\left(\tau e^{-\beta D}+(1-\tau)\right)=e^{-\beta D_{\tau}}\left(e^{-\beta D_{\tau}}+1\right), D_{\tau}=D-\beta^{-1} \log \tau /(1-\tau)$. In particular, taking $\beta=0$, the restriction of the trace on $A(\Gamma)$ to $\mathcal{O}$ is the restriction of the Powers state $\phi$, on $\operatorname{Cliff}_{\mathbb{C}}\left(\ell^{2}(\mathbb{Z})_{\mathbb{R}}\right)$, for $\lambda=\tau /(1-\tau)$.

Next we show that there exists a projection of norm one from $A(\Gamma)$ onto the observable algebra $j(\mathcal{O})$.

Lemma 5.2. Let $\Gamma$ be a connected graph. Consider the type $I_{1}$ representation associated with the trace $\operatorname{Tr}$ on $A(\Gamma)$, and $E$ the orthogonal projection on $j(O)$. Then $E(x) \in j \mathcal{O}[-2 m, 2 m]$, for all $x \in A^{\Gamma}(-2 m, 2 m)$, if we let $\mathcal{O}(\Lambda)=A^{1 / 2}(\Lambda)^{\mathbb{T}}$.

Proof. We have $x y=y x$ for all $x \in A(-2 m, 2 m), y \in \mathcal{O}((-\infty,-2 m-1]$ $\cup[2 m+1, \infty))$. Hence $E(x) y=E(x y)=E(y x)=y E(x)$ for all $x \in A(-2 m, 2 m)$, $y \in \mathcal{O}((-\infty,-2 m-1] \cup[2 m+1, \infty))$. Consequently, if $R_{\lambda}(\Lambda)$ denotes the weak closure of $A^{1 / 2}(\Lambda)$ in the Powers state $\phi_{\lambda}$, and $R_{\lambda}^{\phi}(\Lambda)$ denotes the centraliser $\left(A^{1 / 2}(\Lambda)^{\mathbb{T}}\right)^{\prime \prime}$, then

$$
\begin{aligned}
E(x) \in R_{\lambda}(-\infty, 2 m-1] & \otimes R_{\lambda}[-2 m, 2 m] \otimes R_{\lambda}[2 m+1, \infty) \cap R_{\lambda}^{\phi}(-\infty, 2 m+1] \\
& \otimes \mathbb{C} \otimes R_{\lambda}^{\phi}[2 m+1, \infty) \subset R_{\lambda}[-2 m, 2 m] .
\end{aligned}
$$

To calculate $\omega_{\beta}$, take $x \in \mathscr{A}_{n}, m \geqq n$, then

$$
\omega_{\beta}(x)=\operatorname{Tr}\left(e^{-\beta H_{m}} x\right) / \operatorname{Tr}\left(e^{-\beta H_{m}}\right)=\operatorname{Tr} e^{-\beta H_{m}} E(x) / \operatorname{Tr}\left(e^{-\beta H_{m}}\right)=\omega_{\beta}(E(x))
$$

using $H_{m} \in j(\mathcal{O})$. Factoriality is clear, by uniqueness of KMS states for $\sigma_{t}$. Now $\omega_{\beta}\left(e_{2 n}\right) \rightarrow 0$ as $n \rightarrow \infty$, and $\omega_{\beta}\left(1-e_{2 n}\right) \rightarrow 0$ as $n \rightarrow-\infty$. Thus it is convenient to introduce the projection in $\mathscr{M}=A(\Gamma)^{\prime \prime}$ given by

$$
f=\lim _{N \rightarrow \infty} e_{-2 N} e_{-2 N+2} \ldots e_{-4} e_{-2}\left(1-e_{2}\right)\left(1-e_{4}\right) \ldots\left(1-e_{2 N}\right)
$$


The automorphism group $\sigma_{t}$ extends to a weakly continuous one parameter group of $*$-automorphism of $\mathscr{M}$. For $x \in A_{m}, N \geqq m$,

$$
\begin{aligned}
\sigma_{t}(f x f) & =\lim _{N \rightarrow \infty} \sigma_{t}\left(f_{N} x f_{N}\right)=\lim _{N \rightarrow \infty} \operatorname{Ad}\left(\exp i t H_{N}\right)\left(f_{N} x f_{N}\right) \\
& =\lim _{N \rightarrow \infty} f_{N} x f_{N}=f x f \quad \text { as } \quad\left[H_{N}, f_{N} x f_{N}\right]=0 .
\end{aligned}
$$

Consequently, $\omega_{\beta} \mid \mathscr{M}_{f}$ is a trace. But

$$
\sigma\left(f\left(1-e_{0}\right)=f e_{0}, \quad \text { and } \quad \omega_{\beta}\left(f e_{0}\right) / \omega_{\beta}\left(f\left(1-e_{0}\right)\right)=\tau /(1-\tau) .\right.
$$

This means that $\mathscr{M}$ is not of type $\mathrm{II}_{1}$ for if $\phi$ is then the trace of $\mathscr{M}, \phi \circ \sigma=\phi$ and $\phi \mid \mathscr{M}_{f}=\omega_{\beta}$ would contradict (5.4). Note moreover that

$$
\sigma^{n}\left(f\left(1-e_{0}\right)\right)=\ldots e_{-2 N} \ldots e_{0} e_{2} \ldots e_{2 n}\left(1-e_{2 n+2}\right) \ldots,
$$

$n=0,1,2, \ldots$ are a family of orthogonal projections whose sum

$$
\sum_{n=0}^{\infty} \sigma^{n}\left(f\left(1-e_{0}\right)\right)=\ldots e_{-2 N} \ldots e_{-4} e_{2} e_{4} \ldots=e
$$

has finite trace. Thus $(A(\Gamma))_{e}^{\prime \prime}$ is a factor of type $\mathrm{II}_{1}$. On the other hand note that $A(\Gamma)_{e}$ can be identified with $A^{T}[0, \infty)$.

\section{Implementation of the Action of the Current Algebra at Zero Temperature}

Let $\Gamma$ be a connected graph, with Jones' index $\tau^{-1}$, and $j$ an embedding of the observable algebra $\mathcal{C}$ in $A^{T}$ as in Theorem 4.3. We first seek a canonical ground state for $L_{0}$. In analogy with what was done in the Fermion algebra (see the appendix), we consider the dynamics $L_{0}^{\varepsilon}=L_{0}+\varepsilon \delta_{0}$ corresponding to the perturbation $D+\varepsilon$ of the Dirac Hamiltonian, where $\delta_{k}$ is the derivation

$$
\delta_{k}(x)=\sum_{n} i\left[j\left(a_{n+k}^{*} a_{n}\right), x\right],
$$

defined on the elements $x$ of finite support in $A^{\Gamma}$. The derivations $\delta_{k}$ leave the Jones' algebra $A^{\tau}$ and the observable algebra $j(\mathcal{O})$ invariant. Then $L_{0}^{\varepsilon}$ has an unique KMS state $\omega_{\beta}^{\varepsilon}$ at inverse temperature $\beta$ with

$$
\omega_{\beta}^{\varepsilon}(x)=\operatorname{Tr} e^{-\beta H_{m}^{\varepsilon}} x / \operatorname{Tr} e^{-\beta H_{m}^{\varepsilon}}
$$

for $x \in A^{\Gamma}[-2 m, 2 m]$, if $H_{m}^{\varepsilon}=\sum_{|n| \leqq m}(n+\varepsilon) e_{2 n}$.
Define

$$
\omega_{\infty}(x)=\lim _{\varepsilon \downarrow 0} \lim _{\beta \rightarrow \infty} \omega_{\beta}^{\varepsilon}(x)=\lim _{N \rightarrow \infty} \operatorname{Tr}\left(f_{N} x\right) / \operatorname{Tr} f_{N}
$$

for $x \in A^{T}$, where $f_{N}=e_{-2 N} \ldots e_{-4} e_{-2}\left(1-e_{0}\right)\left(1-e_{2}\right) \ldots\left(1-e_{2 N}\right)$. Then $\omega_{\infty}$ is a ground state for $L_{0}$ on $A^{\tau}$, and $\omega_{\infty}\left|\mathcal{O}=\omega_{A^{\infty}}\right| \mathcal{O}$ (for any embedding $j$ of the observable algebra in $A^{\Gamma}$ ), where $\omega_{A^{\infty}}$ is the ground state (Appendix) of $L_{0} \mid \mathcal{O}$.

For $f \in C\left(S^{1}, \mathbb{R}\right)$ consider the potential function $\Phi=\Phi^{f}$ defined on finite subsets $X \subset \mathbb{Z}$ by

$$
\Phi(X)=\hat{f}_{n} j\left(a_{m+n}^{*} a_{m}\right)+\hat{f}_{-n} j\left(a_{m}^{*} a_{n+m}\right)
$$


if $X=\{m, m+1, \ldots, m+n\}, m \in \mathbb{Z}, n \in \mathbb{N}$, and $\Phi(X)=0$ otherwise. Let

$$
\mathscr{C}=\left\{f \in C\left(S^{1}, \mathbb{R}\right): \alpha=\alpha_{f}^{\lambda}=2 \sum_{n=0}^{\infty} e^{\lambda n}(n+1) \hat{f}_{n+1}<\infty \text { for some } \lambda>0\right\},
$$

and for $f \in \mathscr{C}$, construct the derivation $J(f)$ on the local elements $A_{0}$ in $A^{\Gamma}$, using the potential $\Phi^{f}$ defined above.

Thus

$$
J(f)(x)=\sum_{m, n} i\left[\hat{f}_{n} j\left(a_{m+n}^{*} a_{m}\right), x\right], x \in A_{0},
$$

and by [BR, Theorem 6.2.4], $A_{0}$ consists of analytic elements for $J(f), J(f)$ is closable and its closure, also denoted by $J(f)$ generates a strongly continuous oneparameter group of *-automorphisms $\theta_{t}^{f}$ of $A^{\Gamma}$ such that

$$
\begin{aligned}
\theta_{t}^{f}(x) & =\sum_{n=0}^{\infty}(J(f))^{n}(x) / n !, & & x \in A_{0} \\
& =\lim _{n \rightarrow \infty} \operatorname{Ad}\left(\exp \left(i H_{n}^{f} t\right)\right)(x), & & x \in A,
\end{aligned}
$$

where $H_{n}^{f}=\sum_{|\alpha|,|\beta| \leqq n} \hat{f}_{\alpha-\beta} j\left(a_{\alpha}^{*} a_{\beta}\right)$.

Moreover, by [BR, Theorem 6.2.4],

$$
\left\|J(f)^{n}(x)\right\| \leqq\left(2 \alpha_{f}^{\lambda} / \lambda\right)^{n}\|x\| n ! \exp \lambda(2 m+1)
$$

for $x \in A[-2 m, 2 m]$, and it is clear from (6.7) that $\theta_{t}^{f} \theta_{t}^{g}=\theta_{t}^{f+g}, f, g \in \mathscr{C}, t \in \mathbb{R}$.

Let $\left(\pi_{\infty}, K, \Omega\right)$ denote the GNS triple of $\omega_{\infty}$ on $A^{\Gamma}$. For $f \in \mathscr{C}$, let

$$
\mathscr{D}(: J(f):)=\left\{\xi \in K: \lim _{N \rightarrow \infty} \sum_{|\alpha|,|\beta| \leqq n} \hat{f}_{\alpha-\beta} \pi_{\infty} j\left(: a_{\alpha}^{*} a_{\beta}:\right) \xi \text { exists }\right\},
$$

and let

$$
: J(f): \xi=\lim _{N \rightarrow \infty} \sum_{|\alpha|,|\beta| \leqq n} \hat{f}_{\alpha-\beta} \pi_{\infty} j\left(: a_{\alpha}^{*} a_{\beta}:\right) \quad \text { for } \quad \xi \in \mathscr{D}(: J(f):) .
$$

Then $x \Omega \in \mathscr{D}(: J(f):)$ for all $x \in A_{0}$, as

$$
\lim _{N \rightarrow \infty} \sum \hat{f}_{\alpha-\beta} \pi_{\infty} j\left(: a_{\alpha}^{*} a_{\beta}:\right) \Omega \text { in the Hilbert space }\left[\pi_{\infty} j(\mathcal{O}) \Omega\right]^{-}
$$

exists [and is :J(f):S] by the classical theory in the appendix, and

$$
J(f)(x)=\lim _{n \rightarrow \infty} \sum_{|\alpha|,|\beta| \leqq n} i\left[\hat{f}_{\alpha-\beta} j\left(: a_{\alpha}^{*} a_{\beta}:\right), x\right]
$$

by (6.6). Consequently for $x \in A_{0}$ :

$$
i: J(f): x \Omega=J(f)(x) \Omega+i x: J(f): \Omega .
$$

Inductively we see that $A_{0} \Omega \subset \mathscr{D}\left((: J(f):)^{n}\right)$ and

$$
(i: J(f):)^{n} x \Omega=\sum_{r=0}^{n}{ }^{n} C_{r} J(f)^{n-r}(x)(i: J(f):)^{r} \Omega .
$$


Hence by (6.9) and (Appendix), for $x \in \mathscr{A}[-2 m, 2 m]$ :

$$
\begin{aligned}
& \|: J(f):)^{n} x \Omega \| \\
& \quad \leqq \sum_{r=0}^{n}{ }^{n} C_{n}\left(2 \alpha_{f}^{\lambda} / \lambda\right)^{n-r}\|x\|(n-r) ! \exp \lambda(2 m+1)((2 r) ! / r !)^{1 / 2}(|f| / 2)^{r} \\
& \quad=\sum_{r=0}^{n}\left(2 \alpha_{f}^{\lambda} / \lambda\right)^{n-r}((2 r) !)^{1 / 2}(r !)^{-3 / 2}(|f| / 2)^{r} n !\|x\| \exp \lambda(2 m+1)
\end{aligned}
$$

Thus

$$
\begin{aligned}
& \sum_{n=0}^{\infty} t^{n}\left\|(: J(f):)^{n} x \Omega\right\| / n ! \\
& \quad \leqq\|x\| \exp \lambda(2 m+1) \sum_{r=0}^{\infty}(t|f| / 2)^{r}(2 r) !^{1 / 2}(r !)^{-3 / 2} \sum_{n=r}^{\infty}\left(2 t \alpha_{f}^{\lambda} / \lambda\right)^{n-r} \\
& \quad=\|x\| \exp \lambda(2 m+1)\left(1-2 t \alpha_{f}^{\lambda} / \lambda\right)^{-1} \sum_{r=0}^{\infty}(t|f| / 2)^{r}(2 r) !^{-1 / 2}(r !)^{-3 / 2},
\end{aligned}
$$

$<\infty$ for sufficiently small $t,\left(2|t| \alpha_{f}^{\lambda} / \lambda<1\right)$.

Hence the symmetric operator: $J(f)$ : has a dense set of analytic elements and so is essentially self adjoint. We again denote its closure by : $J(f)$ :.

The derivations $J(f)$ and $J(g)$ commute (for $f, g \in \mathscr{C}$ ). Thus for $x \in A_{0}$, we have

$$
[[i: J(f):, i: J(g):], x] \Omega=[[J(f), J(g)], x] \Omega=0 .
$$

Hence

$$
[: J(f):,: J(g):] \times \Omega=x[: J(f):, J(g):] \Omega=x \frac{1}{2 \pi i} \int f d g \Omega
$$

where in the last equality we have used the classical theory of the Appendix in the Hilbert space $\left[\pi_{\infty} j(\mathcal{O}) \Omega\right]^{-}$.

We summarize this as follows, using the notation

$$
\tilde{U}\left(C^{\infty}\left(S^{1}\right)\right)=\left\{h \in U\left(C^{\infty}\left(S^{1}\right)\right): h=U^{m} \exp i g, m \in \mathbb{Z}, g \in \mathscr{C}\right\} .
$$

Theorem 6.1. Let $\Gamma$ be a connected graph with Jones index $\tau^{-1}$.

(6.18) $J(f)=\sum_{-\infty}^{\infty} \hat{f}_{n} \delta_{n}, f \in \mathscr{C}$ defines a distribution with values in the derivations of $A^{I}$ (respectively $\left.A^{\tau}\right)$ and a representation of $\tilde{U}\left(C^{\infty}\left(S^{\prime}\right)\right)$ in the group of automorphisms of $A^{I}$ (respectively $A^{\tau}$ ).

(6.19) The time evolution $\sigma_{t}$ satisfies $J(\dot{f})=-J\left(f^{\prime}\right)$.

(6.20) The action of the current algebra leaves the state $\omega_{\infty}$ :

$$
\omega_{\infty}(x)=\lim _{N \rightarrow \infty} \frac{\operatorname{Tr}\left(e_{-N} \ldots e_{-2}\left(1-e_{0}\right) \ldots\left(1-e_{N}\right) x\right)}{\operatorname{Tr}\left(e_{-N} \ldots e_{-2}\left(1-e_{0}\right) \ldots\left(1-e_{N}\right)\right.}
$$

on $A^{\Gamma}$ (respectively $A^{\tau}$ ) quasi-invariant, and is implemented by self adjoint operators $: J(f):(f \in \mathscr{C})$ satisfying

$$
[: J(f):, J(g):]=\frac{1}{2 \pi i} \int f d g
$$


To get a representation of the Virasoro algebra, with $c=1$, from the currents $T_{n}=: J_{n}$ :, one uses the Sugawara formula:

$$
L_{n}=\sum \frac{1}{2} \circ T_{m+n} T_{-m} \circ
$$

where

$$
{ }_{\circ}^{\circ} T_{m} T_{n \circ}^{\circ}=\left\{\begin{array}{lll}
T_{m} T_{n} & \text { if } & m<0 . \\
T_{n} T_{m} & & m>0 .
\end{array}\right.
$$

\section{Appendix}

We recall the Fermi-Dirac quantisation of the classical fields on $S^{1}$. If $H$ is a real Hilbert space, the Clifford algebra $\operatorname{Cliff}_{\mathbb{C}}(H)$ is the universal $C^{*}$-algebra generated by the range of a linear map $\gamma$ on $H$ with the relations $\gamma(h)^{*}=\gamma(h), \gamma(h)^{2}=\|h\|^{2}$, $h \in H$. Cliff $\mathbb{C}_{\mathbb{C}}(H)$ has a $\mathbb{Z} / 2$ grading so that each $\gamma(h)$ is odd. If $i$ is a complex structure on $H$ then $a(f)=[\gamma(f)-i \gamma(i f)] / 2$ for $f \in H_{\mathbb{C}}$, the complexification of $H$, defines a representation of the canonical anti-commutation relations. If $\left\{e_{i}\right\}_{i \in I}$ is a complete orthonormal basis for $H_{\mathbb{C}}$, we let $a_{i}=a\left(e_{i}\right)$. The field algebra $\mathscr{A}$ is Cliff $\mathbb{C}\left(\ell^{2}(\mathbb{Z})_{\mathbb{R}}\right)$, and $\psi(\theta)=\sum a_{n} e^{-i n \theta}$ gives the Fermi quantum field.

The gauge group $U(1)$ of the first kind acts on $\mathscr{A}$ by $\alpha_{s}\left(a_{n}\right)=e^{-i s} a_{n}$. The corresponding derivation $\delta=\left(\alpha_{s}\right)_{s=0}^{\prime}$ takes $a_{n}$ to $-i a_{n}$, and is given by $\delta(x)$ $=i \sum\left[a_{n}^{*} a_{n}, x\right]$. The algebra of observable $\mathcal{O}$ is the $\alpha$-fixed point algebra, and is generated by $\left\{a_{n}^{*} a_{m}, 1\right\}$.

The gauge group $U\left(C\left(S^{1}\right)\right)$ of the second kind acts on $\mathscr{A}$ by $f: \psi(h) \rightarrow \psi(f h)$. Moreover $C^{\infty}\left(S^{1}, U(1)\right)\left(C C\left(S^{1}, U(1)\right)=U\left(C\left(S^{1}\right)\right)\right)$ is generated by the shift $U$ and the Lie algebra of real valued smooth maps on $S^{1}$, in that we can write any $f \in C^{\infty}\left(S^{1}, U(1)\right)$ uniquely as $f=U^{m} f_{0}$, where $m$ is the winding number of $f$ and $f_{0}=\exp i g$ for some $g \in C^{\infty}\left(S^{1}, \mathbb{R}\right)$. Then $g \in C^{\infty}\left(S^{1}, \mathbb{R}\right)$ gives a $*$-derivation $J(g)$ on $\mathscr{A}_{0}$ the $*$-algebra generated by $\left\{a_{m}\right\}$. Complexifying, and taking $g=e^{i m \theta}$, there is a derivation $\delta_{m}$ such that $\delta_{m}\left(a_{n}\right)=-i a_{n+m}, \delta_{m}\left(a_{n}^{*}\right)=i a_{n-m}^{*}$. Then $\delta_{m}^{*}=\delta_{-m}$, and

$$
\delta_{m}(x)=i \sum_{n}\left[a_{n+m}^{*} a_{m}, x\right]
$$

For $g=\sum \hat{g}_{m} e^{i m \theta}, J(g)=\sum \hat{g}_{m} \delta_{m}=\frac{1}{2 \pi} \int f(\theta) J(\theta) d \theta$, where $J(\theta)=\sum e^{-i m \theta} \delta_{m}$ is a distribution taking values in the derivations on $\mathscr{A}_{0}$.

Time evolution is given by $\sigma_{t}=\operatorname{Cliff}\left(e^{i t D}\right)$ on $\mathscr{A}$, where $D$ is the Dirac operator $-\mathrm{id} / d \theta$ on $S^{1}$, or $\sigma_{t}\left(a_{n}\right)=e^{- \text {int }} a_{n}$. The $*$-derivation $L_{0}=\left(\sigma_{t}\right)_{t=0}^{\prime}$ takes $a_{n}$ to in $a_{n}$ and

$$
L_{0}(x)=\sum \operatorname{in}\left[a_{n}^{*} a_{n}, x\right] \text {. }
$$

Conservation of the current is expressed by $\left[L_{0}, J(\theta)\right]=-J^{\prime}(\theta)$.

If $C$ is a positive contraction on a complex Hilbert space $K$, we let $\omega_{C}$ denote the quasi-free state on $\operatorname{Cliff}_{\mathbb{C}}\left(K_{\mathbb{R}}\right)$ such that $\omega\left(a^{*}(f) a(g)\right)=\langle C f, g\rangle$. If $D$ is a self-adjoint operator, then for all $0 \leqq \beta<\infty$, there exists an unique KMS state $\omega_{\beta}$ at inverse temperature $\beta$ for $\sigma_{t}=\operatorname{Cliff}\left(e^{i t D}\right)$, which is quasi-free with covariance operator $A_{\beta}=e^{--\beta D}\left(e^{-\beta D}+1\right)^{-1}$. The Dirac Hamiltonian has a kernel, there is no unique 
ground state, so we take the canonical ground state $\omega_{\infty}$ with covariance operator

$$
A_{\infty}=\lim _{\varepsilon \downarrow 0} \lim _{\beta \rightarrow \infty} e^{-\beta(D+\varepsilon)}\left[e^{-\beta(D+\varepsilon)}+1\right]^{-1}=(1-F) / 2,
$$

where $F=\lim _{\varepsilon \downarrow 0}(D+\varepsilon)|D+\varepsilon|^{-1}$ is the "phase" of $D$. In the state $\omega_{\infty}$ using the normal ordering $: a_{i}^{*} a_{j}:=a_{i}^{*} a_{j}-\omega_{\infty}\left(a_{i}^{*} a_{j}\right), L_{0}$ is implemented by $\sum n: a_{n}^{*} a_{n}$ : and $\delta_{k}$ by $J_{k}=\sum: a_{n+k}^{*} a_{n}:$ Then $J_{k}^{*}=J_{-k}$, and $\left[J_{m}, J_{n}\right]=n \delta_{m .-n}$. Consequently $J(g)$ is implemented by $: J(g):=\sum \hat{\mathrm{g}}_{m} J_{m}$, and

$$
[: J(f):,: J(g):]=\frac{1}{2 \pi i} \int_{S^{1}} f d g
$$

for $f, g \in \mathscr{A}=\left\{h \in C\left(S^{1}\right):|h|^{2} \equiv \sum|k|^{2}\left|\hat{h}_{k}\right|^{2}<\infty\right\}$. Thus $f \rightarrow \exp i: J(f)$ : is a representation of the canonical commutation relations on $\mathscr{A}$. Moreover, the vacuum vector $\Omega$ gives the Boson Fock state

$$
\langle\exp i: J(f): \Omega, \Omega\rangle=\exp -|f|^{2} / 4 \text {. }
$$

Thus $f \rightarrow: J(f)$ : is a free Bose field and

$$
\left\langle: J(f):^{2 n} \Omega, \Omega\right\rangle=\frac{(2 n) !}{n !}\left(\frac{|f|}{2}\right)^{2 n} .
$$

\section{References}

[ABF] Andrews, G.E., Baxter, R.J., Forrester, J.P.: Eight vertex SOS model and generalised Rogers-Ramanujan type identities. J. Stat. Phys. 35, 193-266 (1984)

[A] Araki, H.: Analyticity of correlation functions for the two dimensional Ising model. Commun. Math. Phys. 106, 241-266 (1986)

[AE] Araki, H., Evans, D.E.: On a $C^{*}$-algebra approach to phase transition in the twodimensional Ising model. Commun. Math. Phys. 91, 489-503 (1983)

[B] Baxter, R.J.: Exactly solved models in statistical mechanics. London: Academic Press 1982

[BPZ] Belavin, A.A., Polyakov, A.M., Zamolodchikov, A.B.: Infinite conformal symmetry in two dimensional quantum field theory. Nucl. Phys. B 241, 333-380 (1980)

[BR] Bratteli, O., Robinson, D.W.: Operator algebras and quantum statistical mechanics, Vol. II. Berlin, Heidelberg, New York: Springer 1981

[CE] Carey, A.L., Evans, D.E.: The operator algebras of the two-dimensional Ising model. In: Braids, Libgober, A., Birman, J. (eds.) Contemporary Mathematics 1988

[C] Connes, A.: Non-commutative differential geometry. Publ. IHES 62, 41-144 (1989)

[CK] Connes, A., Karoubi, M.: Caractere multiplicatif d'un module de Fredholm. J. K Theory 2, 431-463 (1988)

[CR] Carey, A.L., Ruisjensaars, S.N.M.: On fermion gauge groups, current algebras, and KacMoody algebras. Acta Appl. Math. 10, 1-86 (1987)

[E] Effros, E.G.: Dimensions and $C^{*}$-algebras, CBMS Lectures 46 Am. Math. Soc. 1981

[EL] Evans, D.E., Lewis, J.T.: On a $C^{*}$-algebra approach to phase transition in the twodimensional Ising model. II. Commun. Math. Phys. 102, 521-535 (1986)

[FQS] Friedan, D., Qiu, D., Shenker, S.: Conformal invariance, unitarity and two dimensional critical exponents. In: Vertex operators in mathematics and physics. Lepowsky, J., Mandelstam, S., Singer, I.M. (eds.), pp. 419-437. Publ. MSRI No. 3. Berlin, Heidelberg, New York: Springer 1984

[GKO] Goddard, P., Kent, A., Olive, D.: Unitary representations of the Virasoro and SuperVirasoro algebras. Commun. Math. Phys. 103, 105-119 (1986) 
[H] Huse, D.: Exact exponents for infinitely many new critical points. Phys. Rev. B 30, 3908-3915 (1984)

[J] Jones, V.F.R.: Index for subfactors. Invent. Math. 72, 1-25 (1983)

[K] Kuik, R.: On the $q$-state Potts model by means of non-commutative algebras. Thesis, Groningen, 1986

[KAW] Kuniba, A., Akutsu, Y., Wadati, M.: Virasoro algebra, von Neumann algebra and critical eight vertex SOS model. J. Phys. Sci. Jpn. 55, 3285-3288 (1986)

[O] Ocneanu, A.: Quantized groups, string algebras and Galois theory for algebras. In Operator Algebras and Applications, Volume II. Proceedings of UK-US Joint Seminar on Operator Algebras. Evans, D.E., Takesaki, M. (eds.). London Math. Soc. Lecture Notes. Cambridge Univ. Press (to appear)

[P] Pasquier, V.: Two dimensional critical systems labelled by Dynkin diagrams, Preprint Saclay 1986

[Pi] Pirogov, S.: States associated with the two dimensional Ising model. Theor. Math. Phys. 11, 614-617 (1972)

[Po] Polyakov, A.M.: Critical symmetry of critical fluctuations. JEPT Lett. 12,381-390(1980)

[PT1] Palmer, J., Tracy, C.: Two dimensional Ising correlations: convergence of the scaling limit. Adv. Appl. Math. 2, 329-388 (1981)

[PT2] Palmer, J., Tracy, C.: Two dimensional lsing correlations: the SMJ analysis. Adv. Appl. Math. 4, 46-102 (1983)

[R] Renault, J.: A groupoid approach to $C^{*}$-algebras. Lecture Notes in Mathematics, Vol. 793. Berlin, Heidelberg, New York: Springer 1980

[SMJ] Sato, M., Miwa, T., Jimbo, M.: Holonomic quantum fields. V. Publ. RIMS Kyoto 16, $531-584(1980)$

[SO'C] Schor, R., O'Carroll, M.: The scaling limit and Osterwalder-Schrader axioms for the two dimensional Ising model. Commun. Math. Phys. 84, 153-170 (1982)

[TL] Temperley, H.N.V., Lieb, E.: Relations between the "percolation" and "colouring" problem and other graph theoretical problems associated with regular planar lattices: Some exact results for the "percolation" problem. Proc. R. Soc. London A 322, 251-280 (1971)

Communicated by A. Jaffe

Received September 26, 1988 
